\title{
CONVOLUTION AND FRACTIONAL INTEGRATION WITH MEASURES ON HOMOGENEOUS CURVES IN $\mathbb{R}^{n}$
}

\author{
Philip Gressman
}

\section{Introduction}

In this paper we consider convolution operators on $\mathbb{R}^{n}$ of the form

$$
R_{\gamma} f(x):=\int_{-1}^{1} f(x-h(t))|t|^{-1+\gamma} d t
$$

where $h(t):=\left(t^{d_{1}}, \ldots, t^{d_{n}}\right)$ for a strictly increasing sequence of positive integers $d_{1}, \ldots, d_{n}$ and $0<\gamma \leq 1$. Let $p_{0}:=|d| /(\gamma n)$ and $q_{0}:=|d| /(\gamma(n-1))$, where $|d|:=\sum_{j} d_{j}$. Let $\mathcal{T} \subset[0,1] \times[0,1]$ be the closed trapezoid with vertices $(0,0)$, $(1,1), \mathcal{Q}:=\left(p_{0}^{-1}, q_{0}^{-1}\right)$, and $\mathcal{Q}^{\prime}:=\left(1-q_{0}^{-1}, 1-p_{0}^{-1}\right)$. We will show that $R_{\gamma}$ is bounded from $L^{p}\left(\mathbb{R}^{n}\right)$ to $L^{q}\left(\mathbb{R}^{n}\right)$ if the point $\left(p^{-1}, q^{-1}\right)$ is in $\mathcal{T} \backslash\left\{\mathcal{Q}, \mathcal{Q}^{\prime}\right\}$. This result is optimal, except possibly at $\mathcal{Q}$ and $\mathcal{Q}^{\prime}$ (i.e., $R_{\gamma}$ is not bounded when $\left.\left(p^{-1}, q^{-1}\right) \notin \mathcal{T}\right)$. In the place of strong estimates at these exceptional points, we will prove restricted weak type estimates.

The method of proof we use was first employed by Christ in [1] to study the case of the curve $h(t)=\left(t, t^{2}, \cdots, t^{n}\right)$ with no fractional integration $(\gamma=$ 1). Tao and Wright have established strong $(p, q)$ boundedness for completely general families of smooth curves [9], but in that paper they were unable to show boundedness in endpoint cases. Earlier papers, such as Oberlin [5], [6] and Greenleaf, Seeger, and Wainger [3], have attained boundary estimates (even strong boundary estimates) but are limited to dimensions $n \leq 4$, with work in dimension 4 restricted to the curve $h(t)=\left(t, t^{2}, t^{3}, t^{4}\right)$.

\section{Preliminaries and the Parameter Sets $\Omega_{k}$}

To prove the desired estimates, it suffices to consider a truncated version of (1) in which $t$ ranges from 0 to 1 . The corresponding half $-1 \leq t \leq 0$ can be treated analogously. Also it suffices (by duality and interpolation) to prove $R_{\gamma}$ satisfies a restricted weak type $\left(p_{0}, q_{0}\right)$ estimate. Furthermore, we will take $\gamma=1$ throughout most of the paper (the case $0<\gamma<1$ will be deduced from the case $\gamma=1$ by interpolation). We have thus reduced matters to the study of the operator $R f(x):=\int_{0}^{1} f(x-h(t)) d t$.

Received January 23, 2004. 
To establish the particular restricted weak type estimate of interest, it is necessary and sufficient to show that for any Borel set $E \subset \mathbb{R}^{n}$ of finite, nonzero measure and any $\alpha>0$

$$
\left|\left\{x \in \mathbb{R}^{n} \mid R \chi_{E}(x)>\alpha\right\}\right| \lesssim\left(|E|^{n} \alpha^{-|d|}\right)^{1 /(n-1)}
$$

(throughout, $u \lesssim v$ means $u \leq C v$ with $C$ depending only on constants fixed a priori such as $n$, the integers $d_{j}$, and the fractional integration parameter $\gamma$ ). From now on, fix $F:=\left\{x \in \mathbb{R}^{n} \mid 2 \alpha \geq R \chi_{E}(x)>\alpha\right\}$ and $\beta:=\frac{1}{|E|} \int_{F} R \chi_{E}$. Since $\beta / \alpha \sim|F| /|E|$, estimate (2) is equivalent to

$$
\alpha^{|d|}(\beta / \alpha)^{n-1} \lesssim|E|
$$

We come now to the engine which will drive the rest of the proof. This first lemma is almost the same as lemma 1 in Christ [1]; the difference is that here the sets $\Omega_{k} \subset \mathbb{R}_{+}^{k}$ are tailored to avoid the coordinate hyperplanes $\left\{t \in \mathbb{R}_{+}^{k} \mid t_{i}=0\right\}$. This new feature was not necessary in [1], but will be essential for our purposes.

Lemma 1. Let $\nu:=\max \{\alpha / 2, \beta / 12\}$, and let $\Phi_{k}: \mathbb{R}^{k} \rightarrow \mathbb{R}^{n}$ be given by $\Phi_{k}\left(t_{1}, \ldots, t_{k}\right):=h\left(t_{1}\right)-h\left(t_{2}\right)+\ldots+(-1)^{k-1} h\left(t_{k}\right)$ for all $1 \leq k \leq 2 n$. There exists an $x_{0} \in E$, a constant $\epsilon$ depending only on $n$, and sets $\Omega_{k} \subset[\nu, 1]^{k}$, $1 \leq k \leq 2 n$, such that for each even $k$

$$
\begin{gathered}
x_{0}+\Phi_{k}\left(\Omega_{k}\right) \subset E \\
\left(t_{1}, \ldots, t_{k}\right) \in \Omega_{k} \Rightarrow\left(t_{1}, \ldots, t_{k-1}\right) \in \Omega_{k-1} \\
\left(t_{1}, \ldots, t_{k-1}\right) \in \Omega_{k-1} \Rightarrow \alpha \geq \int \chi_{\Omega_{k}}\left(t_{1}, \ldots, t_{k}\right) d t_{k} \geq \epsilon \alpha \\
\left(t_{1}, \ldots, t_{k}\right) \in \Omega_{k} \Rightarrow\left|t_{k}-t_{j}\right| \geq \epsilon \alpha \forall j<k,
\end{gathered}
$$

and for each odd $k$ analogous statements hold, replacing $E$ by $F$ and $\alpha$ by $\beta$.

Proof. Consider the truncated operator $\widetilde{R} f(x):=\int_{\nu}^{1} f(x-h(t)) d t$ and the associated sequences of sets

$$
\begin{gathered}
E_{0}:=E, F_{0}:=F \\
E_{j+1}:=\left\{x \in E_{j} \mid \widetilde{R}^{*} \chi_{F_{j}}(x)>2^{-j-3} \beta\right\} \\
F_{j+1}:=\left\{y \in F_{j} \mid \widetilde{R} \chi_{E_{j+1}}(y)>2^{-j-3} \alpha\right\}
\end{gathered}
$$

(In [1], the original operator $R$ is used instead of $\widetilde{R}$ ). To be of any use, the sets $E_{j}$ and $F_{j}$ must be non-empty; we will establish the stronger property

$$
\left\langle\widetilde{R} \chi_{E_{j}}, \chi_{F_{j}}\right\rangle \geq 2^{-j-1} \beta|E| \forall j \geq 0
$$

by induction. Take $j=0$, and suppose that $\nu=\alpha / 2$. We step through definitions in the usual way:

$$
\left\langle\widetilde{R} \chi_{E}, \chi_{F}\right\rangle \geq\left\langle R \chi_{E}, \chi_{F}\right\rangle-\frac{\alpha}{2}|F| \geq \frac{1}{2}\left\langle R \chi_{E}, \chi_{F}\right\rangle=\frac{1}{2} \beta|E| .
$$


If instead $\nu=\beta / 12$, split $E=E_{s} \cup E_{b}$, where $E_{s}$ is the subset of $E$ on which $R^{*} \chi_{F} \leq \beta / 4$ and $E_{b}$ is the relative complement of $E_{s}$ in $E$. We use the trivial inequality $\left\langle\chi_{E}, R^{*} \chi_{F}\right\rangle \leq \frac{\beta}{4}\left|E_{s}\right|+\left\langle\chi_{E_{b}}, R^{*} \chi_{F}\right\rangle$ to estimate $\left\langle\chi_{E}, \widetilde{R}^{*} \chi_{F}\right\rangle$ from below:

$$
\left\langle\chi_{E}, \widetilde{R}^{*} \chi_{F}\right\rangle \geq \frac{2}{3}\left\langle\chi_{E_{b}}, R^{*} \chi_{F}\right\rangle \geq \frac{2}{3}\left(\left\langle\chi_{E}, R^{*} \chi_{F}\right\rangle-\frac{\beta}{4}\left|E_{s}\right|\right) \geq \frac{1}{2} \beta|E| .
$$

As a consequence, it is again true that $\left\langle\widetilde{R} \chi_{E}, \chi_{F}\right\rangle \geq \frac{1}{2} \beta|E|$. This establishes the truth of (8) when $j=0$. The induction step is straightforward:

$$
\begin{aligned}
\left\langle\widetilde{R} \chi_{E_{j+1}}, \chi_{F_{j+1}}\right\rangle & =\left\langle\widetilde{R} \chi_{E_{j+1}}, \chi_{F_{j}}\right\rangle-\left\langle\widetilde{R} \chi_{E_{j+1}}, \chi_{F_{j} \backslash F_{j+1}}\right\rangle \\
& \geq\left\langle\chi_{E_{j+1}}, \widetilde{R}^{*} \chi_{F_{j}}\right\rangle-2^{-j-3} \alpha|F| \\
& \geq\left\langle\chi_{E_{j}}, \widetilde{R}^{*} \chi_{F_{j}}\right\rangle-\left\langle\chi_{E_{j} \backslash E_{j+1}}, \widetilde{R}^{*} \chi_{F_{j}}\right\rangle-2^{-j-3} \beta|E| \\
& \geq\left\langle\widetilde{R} \chi_{E_{j}}, \chi_{F_{j}}\right\rangle-2^{-j-2} \beta|E| \\
& \geq 2^{-j-2} \beta|E| .
\end{aligned}
$$

This establishes the claim (8).

Now the sets $\Omega_{k}$ may be defined. Fix a point $x_{0}$ anywhere in $E_{n}$. By definition of $E_{n}$ and $\widetilde{R}$,

$$
\left|\left\{t \in[\nu, 1] \mid x_{0}+h(t) \in F_{n-1}\right\}\right|>2^{-n-2} \beta .
$$

We may take $\Omega_{1}$ to be any Borel subset of $\left\{t \in[\nu, 1] \mid x_{0}+h(t) \in F_{n-1}\right\}$ with measure $2^{-n-2} \beta$. The construction of $\Omega_{2}$ proceeds iteratively. For each $t_{1} \in \Omega_{1}$, $x_{0}+h\left(t_{1}\right)$ is in $F_{n-1}$; therefore $\widetilde{R} \chi_{E_{n-1}}\left(x_{0}+h\left(t_{1}\right)\right)>2^{-n-1} \alpha$. As a result, the set

$$
\left\{\left(t_{1}, t_{2}\right) \in \Omega_{1} \times[\nu, 1]\left|x_{0}+\Phi_{2}\left(t_{1}, t_{2}\right) \in E_{n-1},\right| t_{2}-t_{1} \mid>2^{-n-2} \alpha\right\}
$$

must have cross-sections (for each $t_{1} \in \Omega_{1}$ ) of measure at least $2^{-n-2} \alpha$. The set $\Omega_{2}$ is now chosen to be a subset of (9) which has cross-sections of measure precisely $2^{-n-2} \alpha$ for each $t_{1} \in \Omega_{1}$; with a little care this may be done so that $\Omega_{2}$ is Borel. For generic odd $k$, we let $\Omega_{k}$ be a Borel subset of

$$
\left\{t \in \Omega_{k-1} \times[\nu, 1]\left|c_{0}+\Phi_{k}(t) \in F_{n-(k+1) / 2}, \forall j<k\right| t_{k}-t_{j} \mid>\epsilon_{k} \beta\right\}
$$

with cross sections (in the $k$-th direction) of measure $2^{-n-4+(k+1) / 2} \beta$. For $k$ even we take $\Omega_{k}$ from

$$
\left\{t \in \Omega_{k-1} \times[\nu, 1]\left|x_{0}+\Phi_{k}(t) \in E_{n-(k / 2)}, \forall j<k\right| t_{k}-t_{j} \mid>\epsilon_{k} \alpha\right\}
$$

with cross sections of measure $2^{-n-3+(k / 2)} \alpha$. In both cases $\epsilon_{k}$ is suitably chosen to permit our choices. These $\Omega_{k}$ satisfy (4) - (7) by construction. 


\section{An Initial Estimate}

It turns out to be quite easy to bound $|E|$ from below with expressions of the form $\alpha^{|d|}(\beta / \alpha)^{\rho}$. The difficulty is, of course, achieving an optimal $\rho$. This will be accomplished in two stages. The first set of estimates will be optimal in dimensions 2 and 3 , and a second set in the next section will yield optimal exponents for all $n$.

Theorem 1. Let $h(t)$ and $E, F, \alpha, \beta$ be as described above. Then for even $n$

$$
|E| \gtrsim \alpha^{|d|}(\beta / \alpha)^{1+3+\cdots+(n-1)},
$$

and for odd $n$

$$
|E| \gtrsim \alpha^{|d|}(\beta / \alpha)^{-1+|d|-2-4-\cdots-(n-1)} .
$$

Proof. Assume first that $n$ is even. In this case lemma 1 says that $x_{0}+\Phi_{n}\left(\Omega_{n}\right) \subset$ E, so

$$
|E| \geq \int_{\Phi_{n}\left(\Omega_{n}\right)} d x \geq(n !)^{-1} \int_{\Omega_{n}}\left|\operatorname{det}\left(\frac{\partial \Phi_{n}}{\partial t}\right)\right|(t) d t,
$$

using the fact that $\Phi_{n}$ is smooth, and outside a closed, nowhere dense set, the equation $\Phi_{n}(t)=x$ has at most $n$ ! solutions for a given $x$ (this fact will be established shortly).

For $t=\left(t_{1}, \ldots, t_{n}\right) \in \Omega_{n}$, lemma 1 guarantees that $t_{j} \geq \alpha / 2$ for all $j$. Provided $i<j$, lemma 1 also guarantees $\left|t_{j}-t_{i}\right| \gtrsim \alpha$ for $j$ even and $\left|t_{j}-t_{i}\right| \gtrsim \beta$ for $j$ odd. We apply these inequalities to an estimate of the Jacobian determinant (also to be established)

$$
\left|\operatorname{det}\left(\frac{\partial \Phi_{n}}{\partial t}\right)\right|(t) \gtrsim\left(\min _{i}\left|t_{i}\right|\right)^{|d|-n(n+1) / 2} \prod_{i<j}\left|t_{j}-t_{i}\right|,
$$

and conclude $\left|\operatorname{det}\left(\frac{\partial \Phi_{n}}{\partial t}\right)\right| \gtrsim \alpha^{|d|-n}(\beta / \alpha)^{\rho}$ on $\Omega_{n}$, where $\rho=2+4+\cdots+(n-$ 2 ). An induction argument easily establishes $\left|\Omega_{n}\right| \gtrsim \alpha^{n / 2} \beta^{n / 2}$. Applying the estimate of the Jacobian determinant and the estimate of the size of $\left|\Omega_{n}\right|$ to the right-hand side of (12) gives (10).

Consider the case when $n$ is odd. Now $\Phi_{n}\left(\Omega_{n}\right) \subset F$. From (13) and lemma 1 we find $\left|\operatorname{det}\left(\frac{\partial \Phi_{n}}{\partial t}\right)\right| \gtrsim \beta^{|d|+\rho-n(n+1) / 2} \alpha^{\rho-(n-1) / 2}$ on $\Omega_{n}$, where $\rho=2+4+\cdots+$ $(n-1)$ (here we used the fact that $\left.t_{j} \geq \beta / 12\right)$. In this case we also have $\left|\Omega_{n}\right| \gtrsim$ $\alpha^{(n-1) / 2} \beta^{(n+1) / 2}$; proceeding as above we find $|F| \gtrsim \alpha^{|d|}(\beta / \alpha)^{|d|-\rho}$. Multiplying both sides by $(\beta / \alpha)^{-1}$ and recalling $|E| \sim|F|(\beta / \alpha)^{-1}$ gives (11).

Remark. It is worth noting that (11) is dual to the estimate

$$
|E| \gtrsim \alpha^{|d|}(\beta / \alpha)^{2+4+\cdots+(n-1)},
$$

which is of the form found in Christ [1] and gives (3) when $n=3$. Because $R$ and $R^{*}$ are equal (up to conjugation by a reflection), (11) and (14) are equivalent. 
Next we justify making the change of variables $x \mapsto \Phi_{n}(t)$ by demonstrating that almost every $x$ has no more than $n$ ! preimages via $\Phi_{n}^{-1}$. The following lemma establishes this assertion for a broad class of curves $h(t)$.

Lemma 2. Let $h$ be a $C^{1}$ curve in $\mathbb{R}^{n}$ and suppose that

$$
\operatorname{det}\left(\frac{\partial \Phi_{n}}{\partial t}\right)\left(t_{1}, \ldots, t_{n}\right) \neq 0
$$

when $t_{1}, \ldots, t_{n}$ are positive and distinct. Then for any $x \in \mathbb{R}^{n}$, there are no more than $n$ ! points $t$ for which $\Phi_{n}(t)=x$ and $t_{1}, \ldots, t_{n}$ are positive and distinct.

Proof. We will show that $\Phi_{n}$ is one-to-one on the region

$$
\Gamma_{n}:=\left\{\left(t_{1}, \ldots, t_{n}\right) \mid 0<t_{\sigma(1)}<\ldots<t_{\sigma(n)}\right\},
$$

where $\sigma$ is any permutation of $\{1,2, \ldots, n\}$. Let $t, t^{\prime} \in \Gamma_{n}, t \neq t^{\prime}$, and for all $j$ from 1 to $n$ let $I_{j}$ be the smallest closed interval containing both $t_{j}$ and $t_{j}^{\prime}$. By the fundamental theorem of calculus,

$$
\Phi_{n}\left(t^{\prime}\right)-\Phi_{n}(t)=\sum_{j}(-1)^{s_{j}} \int_{I_{j}} \frac{d h}{d t}(u) d u=M\left(t^{\prime}, t\right) v
$$

where $s_{j}=j+1$ if $t_{j}^{\prime} \geq t_{j}\left(s_{j}=j\right.$ otherwise), $M\left(t^{\prime}, t\right)$ is the matrix whose $j$-th column is $(-1)^{s_{j}} \int_{I_{j}} \frac{d h}{d t}(u) d u$, and $v=[1 \cdots 1]^{T}$. We wish to show $\Phi_{n}\left(t^{\prime}\right)-$ $\Phi_{n}(t) \neq 0$; one way to accomplish this is to construct a second matrix $\widetilde{M}\left(t^{\prime}, t\right)$ such that $M\left(t^{\prime}, t\right) v=\widetilde{M}\left(t^{\prime}, t\right) v$ and $\operatorname{det} \widetilde{M}\left(t^{\prime}, t\right) \neq 0$. Consider the function

$$
f(x):=\sum_{j=1}^{n}(-1)^{s_{j}} \chi_{I_{j}}(x) .
$$

Since $t^{\prime} \neq t, f$ is not identically zero (because $t^{\prime}$ and $t$ are in the same ordered region $\Gamma_{n}$ ). Modulo endpoints, there exist pairwise disjoint, open intervals $J_{k}$ and non-zero integers $c_{k}$ so that $f(x)=\sum_{k} c_{k} \chi_{J_{k}}(x)$. As there are $n$ intervals $I_{j}$, we may demand without loss of generality that there be $2 n-1$ intervals $J_{k}$. We may also demand that $J_{k}$ be to the left of $J_{k+1}$ for all $k$.

Consider the integer $p_{k}:=\left|c_{k-1}-c_{k}\right|$. If $p_{k} \neq 0$, there must be at least $p_{k}$ intervals $I_{j_{1}}, I_{j_{2}}, \ldots$ which have an endpoint falling somewhere between $J_{k-1}$ and $J_{k}$. Keeping track of these endpoints shows $\sum_{k} p_{k} \leq 2 n-2$.

If $k_{1}, k_{2}, \ldots, k_{L}$ is any subsequence of $1,2, \ldots,(2 n-1)$ chosen so that $c_{k_{l}}$ alternates in sign, we have $2(L-1) \leq \sum_{l}\left|c_{k_{l}}-c_{k_{l-1}}\right| \leq 2(n-1)$ or $L \leq n$. Thus, the function $f$ cannot be too oscillatory. In particular, we may divide the support of $f$ (again modulo endpoints) into $n$ non-empty, pairwise disjoint intervals $F_{k}$ on which $f$ is either non-negative or non-positive (and never identically zero).

We now define $\widetilde{M}\left(t^{\prime}, t\right)$ by taking its $k$-column to be $\int_{F_{k}} \frac{d h}{d t}(u) f(u) d u$. The condition $M\left(t^{\prime}, t\right) v=\widetilde{M}\left(t^{\prime}, t\right) v$ is an immediate consequence of the fact that 
the $F_{k}$ are disjoint and cover the support of $f$. By multilinearity, taking $u=$ $\left(u_{1}, \ldots, u_{n}\right)$,

$$
\operatorname{det} \widetilde{M}\left(t^{\prime}, t\right)=(-1)^{n-1} \int_{\Pi_{k} F_{k}}\left(\operatorname{det}\left(\frac{\partial \Phi_{n}}{\partial u}\right)(u) \prod_{k=1}^{n} f\left(u_{k}\right)\right) d u \text {. }
$$

The integrand has constant sign and is non-zero on a set of positive measure, so $\operatorname{det} \widetilde{M}\left(t^{\prime}, t\right) \neq 0$.

The final piece of theorem 1 is to examine the Jacobian determinant of $\Phi_{n}(t)$. The following lemma is a well-known fact about Vandermonde-type determinants (see for example pp. 200-201 of [10]; it is also readily established by induction on $n$ ). In particular, (13) and (15) follow immediately.

Lemma 3. Let $h(t)=\left(t^{d_{1}}, \ldots, t^{d_{n}}\right)$, where $1 \leq d_{1}<\cdots<d_{n}$ are integers. Then

$$
\operatorname{det}\left(\frac{\partial \Phi_{n}}{\partial t}\right)(t)=c \mathcal{P}(t) \prod_{i<j}\left(t_{j}-t_{i}\right)
$$

where $\mathcal{P}$ is a symmetric, homogeneous polynomial with positive integer coefficients and the constant $c$ equals $(-1)^{n-1} \prod_{j} d_{j}$.

\section{The Main Estimate}

When $\beta / \alpha<<1$, theorem 1 fails to prove (3). The reason is that lemma 1 does not offer enough control over the distances $\left|t_{i}-t_{j}\right|$ to prove a sufficient lower bound for the Jacobian determinant of $\Phi_{n}$. To resolve this problem we return to the ideas of Christ [1] to gain more control over these distances (for example, taking into account that they are not independent of one another).

For bookkeeping purposes, we will construct a partition $\Lambda$ (hereafter called a band structure following the terminology of [1]) of the indices $\{1,2, \ldots, k\}$ into disjoint subsets (called bands) in such a way that $\left|t_{i}-t_{j}\right|$ is "big" when $i$ and $j$ are in distinct bands and "small" when $i$ and $j$ are in the same band. Taking a cue from lemma 1, we demand that that no two even indices be in the same band and that no even index be in the same band as the index 1 . We will also classify each index $1, \ldots, k$ according to its status within its own band; possible types are free, quasi-free, and bound. The classification proceeds according to the following rules:

1. Each band will have precisely one free index. If a band contains an even index or the index 1, then that index will be free (no band can contain two such indices). Otherwise the lowest index in a band is called free.

2. In any band containing exactly two indices, the index not free is quasifree. A quasi-free index together with its free companion will be called a free/quasi-free pair.

3. All indices not free or quasi-free are bound. A bound index is said to be bound to the unique free index of its band. 
If index $i$ is bound to index $j$, we write $i \Rightarrow j$ and set $B(i):=j$. We will enumerate the bound indices as $b_{1}, b_{2}, \ldots$, and $f_{1}, f_{2}, \ldots$ will be an enumeration of the remaining indices (those either free or quasi-free).

In the next section we will show that there exists $\omega \subset \Omega_{k}$, for some $k$ with $n \leq k \leq 2 n-2$, and a band structure on $\{1, \ldots, k\}$ such that the following are true $\left(t=\left(t_{1}, \ldots, t_{k}\right)\right.$ is any point in $\left.\omega\right)$ :

(i) $|\omega| \sim\left|\Omega_{k}\right|$.

(ii) The total number of indices classified either free or quasi-free is $n$.

(iii) $\left|t_{i}-t_{j}\right| \geq \delta \alpha$ when $i, j$ belong to distinct bands.

(iv) $\epsilon \beta \leq\left|t_{i}-t_{j}\right| \leq \delta \alpha$ when $i, j$ are a free/quasi-free pair.

(v) $\epsilon \beta \leq\left|t_{i}-t_{j}\right| \leq \delta^{\prime} \alpha$ when $i$ is bound to $j$.

(vi) The constants $\epsilon, \delta$, and $\delta^{\prime} / \delta$ are controlled a priori and small relative to unity.

The set $\omega$ takes the place of $\Omega_{n}$ in theorem 1 and allows for optimal results.

Theorem 2. Let $h(t):=\left(t^{d_{1}}, t^{d_{2}}, \cdots, t^{d_{n}}\right)$ and $E, F, \alpha, \beta$ be as defined. Then for $n \geq 2$, estimate (3) holds, i.e., $|E| \gtrsim \alpha^{|d|}(\beta / \alpha)^{n-1}$.

Proof. Let us take $\omega$ for granted. Consider the case when $k$ is even. As in theorem 1, we have the trivial estimate $|E| \geq\left|\Phi_{k}(\omega)\right|$; however, $\omega$ may live in more than $n$ dimensions. Thus, extra care must be taken to compute $\left|\Phi_{k}(\omega)\right|$. Following [1], we make a preliminary change of variables

$$
\begin{aligned}
& \tau_{j}=t_{f_{j}}, \quad 1 \leq j \leq n \\
& s_{i}=t_{b_{i}}-t_{B\left(b_{i}\right)}, \quad 1 \leq i \leq k-n
\end{aligned}
$$

and regard $\Phi_{k}$ as a function of $(\tau, s), G_{s}(\tau):=\Phi_{k}(t)$. The variable $s$ is fixed and the computations proceed with $G_{s}(\tau)$ acting as a map from $\mathbb{R}^{n}$ to itself; in particular, we make the change of variables $x \mapsto G_{s}(\tau)$ and the estimate $\left|\operatorname{det}\left(\frac{\partial G_{s}}{\partial \tau}\right)\right| \gtrsim \alpha^{|d|-n}(\beta / \alpha)^{\mathcal{M}}$ on $\omega(\mathcal{M}$ is the number of quasi-free indices; we will, of course, prove this). The proof proceeds analogously to theorem 1 :

$$
\begin{aligned}
|E| & \geq\left|\left\{G_{s}(\tau) \mid t(\tau, s) \in \omega\right\}\right| \\
& \gtrsim \int_{\{\tau \mid t(\tau, s) \in \omega\}}\left|\operatorname{det}\left(\frac{\partial G_{s}(\tau)}{\partial \tau}\right)\right| d \tau \\
& \gtrsim \alpha^{|d|-n}(\beta / \alpha)^{\mathcal{M}}|\{\tau \mid t(\tau, s) \in \omega\}| .
\end{aligned}
$$

Now we average this lower bound for $|E|$ over the ball $\left\{s \in \mathbb{R}^{k-n}|| s_{i} \mid \leq \delta^{\prime} \alpha \forall i\right\}$; the result is $|E| \gtrsim \alpha^{|d|-k}(\beta / \alpha)^{\mathcal{M}}|\omega|$. When $k$ is even, $|\omega| \sim \alpha^{k}(\beta / \alpha)^{k / 2}$, hence $|E| \gtrsim \alpha^{|d|}(\beta / \alpha)^{\mathcal{M}+k / 2}$. A simple counting argument shows that $\mathcal{M}+k / 2 \leq n-1$. Thanks to theorem 1 we need only consider the case $\beta \lesssim \alpha$, so (3) follows.

When $k$ is odd, we proceed exactly as above, but conclude instead that $|F| \gtrsim$ $\alpha^{|d|}(\beta / \alpha)^{\mathcal{M}+(k+1) / 2}$. This time at least $(k+1) / 2$ indices are free, so $\mathcal{M}+(k+$ $1) / 2 \leq n$. As a result $|F| \gtrsim \alpha^{|d|}(\beta / \alpha)^{n}$ and $|E| \gtrsim \alpha^{|d|}(\beta / \alpha)^{n-1}$ as desired.

As before, the number of preimages $G_{s}^{-1}(\{x\})$ must be bounded if the corresponding change of variables is to be justified. Unfortunately, arguments like 
lemma 2 no longer work. There is hope, but as in Christ [1] it involves Bezout's theorem. To be precise, we consider the homogeneous system (in the variables $\left.\tau_{0}, \tau_{1}, \ldots, \tau_{n}\right)$

$$
0=\left(x_{1} \tau_{0}^{d_{1}}, x_{2} \tau_{0}^{d_{2}}, \ldots, x_{n} \tau_{0}^{d_{n}}\right)-G_{s \tau_{0}}\left(\tau_{1}, \ldots, \tau_{n}\right) .
$$

From page 198 of [7], this system either has an infinite number of solutions in $\mathbb{C P}^{n}$ or exactly $\prod d_{j}$ solutions counting multiplicity. So long as $x$ is not in the image (under $G_{s}$ ) of the set where the Jacobian of $G_{s}$ is degenerate (which must have measure zero), there cannot be an infinite number of solutions. Fixing $\tau_{0}=1$ and restricting to real space, we see that $x=G_{s}(\tau)$ has at most $\prod_{j} d_{j}$ solutions.

The remaining issue to be resolved in this section is to establish a lower bound of the Jacobian determinant of $G_{s}$ on the set $\omega$. Recall

$$
G_{s}(\tau)=\sum_{j=1}^{n}\left[(-1)^{f_{j}-1} h\left(\tau_{j}\right)+\sum_{b_{i} \Rightarrow f_{j}}(-1)^{b_{i}-1} h\left(\tau_{j}+s_{i}\right)\right] .
$$

As bound indices are necessarily odd, $(-1)^{b_{i}-1}=1$ in all cases. Taking partial derivatives yields

$$
\frac{\partial G_{s}}{\partial \tau_{j}}=(-1)^{f_{j}-1} \frac{d h}{d t}\left(\tau_{j}\right)+\sum_{b_{i} \Rightarrow f_{j}} \frac{d h}{d t}\left(\tau_{j}+s_{i}\right) .
$$

When $s=0, \frac{\partial G_{s}}{\partial \tau_{j}}$ is an integer multiple of $\frac{d h}{d t}$; furthermore, it is a non-zero multiple since no free index may have only one bound companion. Let $M_{h}(\tau)$ be the $n \times n$ matrix whose $j$-th column is $\frac{d h}{d t}\left(\tau_{j}\right)$ and set $V_{h}(\tau):=\operatorname{det} M_{h}(\tau)$. By multilinearity we have

$$
\operatorname{det}\left(\frac{\partial G_{s}}{\partial \tau}\right)(\tau)=\sum_{i}(-1)^{\nu(i)} V_{h}\left(\tau+\epsilon_{i}\right)
$$

$\sum_{i}(-1)^{\nu(i)} \neq 0$ follows, once again, from examining the case $s=0$. We recall (16) and factor $V_{h}$. Take, for example, a term from the right-hand side of (17):

$$
V_{h}\left(\tau+\epsilon_{i}\right)=\mathcal{P}\left(\tau+\epsilon_{i}\right) \prod_{j<l}\left(\tau_{l}-\tau_{j}+\left(\epsilon_{i}\right)_{l}-\left(\epsilon_{i}\right)_{j}\right) .
$$

Observe that $\left|\left(\epsilon_{i}\right)_{l}-\left(\epsilon_{i}\right)_{j}\right| \leq 2 \delta^{\prime} \alpha$. If it happens that $\left|\tau_{l}-\tau_{j}\right|<\delta \alpha$, then $\tau_{l}$ and $\tau_{j}$ must correspond (before the linear change of variables) to a free/quasi-free pair, in which case $\left(\epsilon_{i}\right)_{l}=\left(\epsilon_{i}\right)_{j}=0$ since non-zero perturbations arise only from bound indices. Thus, if $\delta^{\prime} / \delta$ is sufficiently small, we have

$$
\left(1-\frac{2 \delta^{\prime}}{\delta}\right)^{\frac{n(n-1)}{2}} \leq \prod_{j<l} \frac{\tau_{l}-\tau_{j}+\left(\epsilon_{i}\right)_{l}-\left(\epsilon_{i}\right)_{j}}{\tau_{l}-\tau_{j}} \leq\left(1+\frac{2 \delta^{\prime}}{\delta}\right)^{\frac{n(n-1)}{2}} .
$$

Furthermore, $\tau_{l} \geq \alpha / 2$ for all $l$, so $\left(1-2 \delta^{\prime}\right) \tau_{l} \leq \tau_{l}+\left(\epsilon_{i}\right)_{l} \leq\left(1+2 \delta^{\prime}\right) \tau_{l}$ and

$$
\left(1-2 \delta^{\prime}\right)^{|d|-\frac{n(n+1)}{2}} \leq \frac{\mathcal{P}\left(\tau+\epsilon_{i}\right)}{\mathcal{P}(\tau)} \leq\left(1+2 \delta^{\prime}\right)^{|d|-\frac{n(n+1)}{2}} .
$$


With apropriate a priori bounds on $\delta$ and $\delta^{\prime} / \delta$ we may combine the above inequalities to conclude

$$
\left|\operatorname{det}\left(\frac{\partial G_{s}}{\partial \tau}\right)(\tau)-V_{h}(\tau) \sum_{i}(-1)^{\nu(i)}\right| \leq \frac{1}{2}\left|V_{h}(\tau)\right| .
$$

On the set $\omega,\left|V_{h}\right| \gtrsim \alpha^{|d|-n}(\beta / \alpha)^{\mathcal{M}}$ because $\left|\tau_{l}\right| \geq \alpha / 2$ for all $l$ and $\left|\tau_{l}-\tau_{j}\right| \geq \delta \alpha$ unless $\tau_{l}$ and $\tau_{j}$ correspond to a free/quasi-free pair. Finally, $\left|\operatorname{det}\left(\frac{\partial G_{s}}{\partial \tau}\right)\right| \sim V_{h}$ on $\omega$, so the estimate is complete.

The last piece of theorem 2 is to demonstrate the existence of $\omega$ and its band structure, which is the task of the next section.

\section{Existence of Band Structures}

Let $\sigma$ be a permutation of $\{1, \ldots, 2 n-2\}$ for which the set $\widetilde{\Omega}_{2 n-2}:=\{t \in$ $\left.\Omega_{2 n-2} \mid t_{\sigma(1)}<\cdots<t_{\sigma(2 n-2)}\right\}$ satisfies $\left|\widetilde{\Omega}_{2 n-2}\right| \geq\left|\Omega_{2 n-2}\right| /(2 n-2)$ !. For all $j<2 n-2$, we restrict the sets $\Omega_{j}$ according to $\sigma$ :

$$
\widetilde{\Omega}_{j}:=\left\{\left(t_{1}, \ldots, t_{j}\right) \in \Omega_{j} \mid \exists\left(t_{j+1}, \ldots, t_{2 n-2}\right) \text { s.t. }\left(t_{1}, \ldots, t_{2 n-2}\right) \in \widetilde{\Omega}_{2 n-2}\right\} .
$$

Observe that $\left|\widetilde{\Omega}_{j}\right| \sim\left|\Omega_{j}\right|$ for all $j$ (when $j$ is even $\left|\Omega_{j-1}\right| \lesssim \frac{1}{\alpha}\left|\Omega_{j}\right| \lesssim \frac{1}{\alpha}\left|\widetilde{\Omega_{j}}\right| \leq$ $\frac{1}{\alpha} \int_{\Omega_{j-1}} \alpha d t=\left|\widetilde{\Omega}_{j-1}\right|$ and similarly with $\beta$ when $j$ is odd). We will work with $\widetilde{\Omega}_{j}$ instead of $\Omega_{j}$ to simplify computations.

Suppose $r$ is an integer no greater than $2 n-2$; let $\mathcal{S}_{r}$ be the power set of $\{2, \ldots, r\}$. For $\Gamma \in \mathcal{S}_{r}$, define $\widetilde{\Omega}_{r}^{\Gamma}:=\left\{t \in \widetilde{\Omega}_{r} \mid t_{\sigma(j)} \geq t_{\sigma(j-1)}+\delta \alpha\right.$ iff $j \in$ $\Gamma, \sigma(j), \sigma(j-1) \leq r\}$. As $\mathcal{S}_{r}$ has at most $2^{r-1}$ elements, there must be at least one $\Gamma$ so that $\left|\widetilde{\Omega}_{r}^{\Gamma}\right| \geq 2^{1-r}\left|\widetilde{\Omega}_{r}\right|$. Given such a $\Gamma:=\left\{L_{2}, \cdots, L_{R}\right\}, 2 \leq L_{2}<\cdots<$ $L_{R} \leq r$, we may construct a band structure on $\widetilde{\Omega}_{r}^{\Gamma}$ :

$$
\begin{aligned}
\{\{\sigma(1), \sigma(2), \ldots, & \left.\sigma\left(L_{2}-1\right)\right\},\left\{\sigma\left(L_{2}\right), \sigma\left(L_{2}+1\right), \ldots, \sigma\left(L_{3}-1\right)\right\} \\
\cdots, & \left.\left\{\sigma\left(L_{R-1}\right), \ldots, \sigma\left(L_{R}-1\right)\right\},\left\{\sigma\left(L_{R}\right), \ldots, \sigma(r)\right\}\right\}
\end{aligned}
$$

For $j<r$, the sets $\widetilde{\Omega}_{j}^{\Gamma}$ also inherit natural band structures: the band structure of $\widetilde{\Omega}_{j}^{\Gamma}$ is the restriction of the partition (18) to a partition of $\{1, \ldots, j\}$. These bands depend on the parameter $\delta$ (the bands will be said to have size $\delta$ ).

Lemma 4. Given $\delta_{0},\left(\delta_{0}^{\prime} / \delta_{0}\right)$ sufficiently small (depending only on $n$ ), there exists $\delta \leq \delta_{0}, \delta^{\prime}:=\delta\left(\delta_{0}^{\prime} / \delta_{0}\right), k \in\{n, \ldots, 2 n-2\}, \omega \subset \Omega_{k}$, and a band structure such that

(i) $|\omega| \sim\left|\Omega_{k}\right|$.

(ii) The number of indices either free or quasi-free is $n$.

(iii) $\left|t_{i}-t_{j}\right| \geq \delta \alpha$ when $i, j$ belong to distinct bands.

(iv) $\epsilon \beta \leq\left|t_{i}-t_{j}\right| \leq \delta \alpha$ when $i, j$ belong to the same band, $i \neq j$.

(v) $\epsilon \beta \leq\left|t_{i}-t_{j}\right| \leq \delta^{\prime} \alpha$ when $i$ is bound to $j$. 
Proof. To begin, let $k_{0}:=2 n-2, \omega:=\widetilde{\Omega}_{2 n-2}$, take $\delta=\delta_{0}$. Construct bands of size $\delta$ for $\omega$ (and restrict $\omega$ to $\widetilde{\Omega}_{k_{0}}^{\Gamma}$ for appropriate $\Gamma$ ). The variables $\mathcal{N}$ and $\mathcal{M}$ will always represent the number of indices designated free and quasi-free, respectively. If $\delta$ is sufficiently small, all even indices and the index 1 will be free thanks to lemma 1. Properties (i), (iii), and (iv) also hold and will remain true at all times. To achieve (ii) and (v), we follow the recipe given in [1]:

1. Construct bands of size $\delta$ for $\omega$ and restrict $\omega$ accordingly. When $k_{0}=$ $2 n-2$, there are at least $n$ free indices, so $\mathcal{N}+\mathcal{M} \geq n$. Stepping through the process below, $\mathcal{N}+\mathcal{M}$ can never fall below $n$. If $\mathcal{N}+\mathcal{M}=n$, go to step 3; otherwise proceed to step 2.

2. Project $\omega$ onto $\widetilde{\Omega}_{k_{0}-1}$ and reset $k_{0}$ to $k_{0}-1$. The new $\omega$ inherits a compatible band structure from the old, and since we removed only one index, $\mathcal{N}+\mathcal{M}$ can decrease by at most one (it could increase by at most one if a bound index becomes quasi-free). Repeat this restriction process for lower and lower $k_{0}$ until $\mathcal{N}+\mathcal{M}=n$ (since $\mathcal{N}+\mathcal{M} \leq k_{0}$, we must reach the goal in a bounded number of repetitions). When this is accomplished, go to step 3.

3. Let $\delta^{\prime}:=\delta\left(\delta_{0}^{\prime} / \delta_{0}\right), \widetilde{\omega}:=\left\{t \in \omega|| t_{i}-t_{j} \mid \leq \delta^{\prime} \alpha \forall i, j i \Rightarrow j\right\}$. If $|\widetilde{\omega}| \geq|\omega| / 2$, the set $\widetilde{\omega}$ satisfies (i)-(v) with $k:=k_{0}$ and the process is complete. If not, continue to step 4 .

4. Replace $\omega$ by $\omega \backslash \widetilde{\omega}$ and $\delta$ by $\delta\left(\delta_{0}^{\prime} / \delta_{0}\right)$. Because the process did not terminate at step 3 , when we construct new bands of size $\delta$ for $\omega$, at least one index which was bound will become free (one of the old bands must break apart). Furthermore, indices which were previously free will remain free, and indices which were quasi-free will remain quasi-free or become free. As a result, $\mathcal{N}+\mathcal{M}$ has increased (it's now strictly greater than $n$ ) and the total number of bound indices has decreased. Go back to step 2.

Each time the process passes through step 4, the number of bound variables decreases. If there are no bound variables, the process will terminate at step 3 (since $\widetilde{\omega}=\omega$ ). Therefore, the process must terminate in a bounded number of steps. The final set $\widetilde{\omega}$ constructed in step 3 is precisely the set satisfying the conditions of the lemma.

\section{Fractional Integration}

We now consider convolutions with added fractional integration as in (1). As promised, an interpolation argument quickly reduces the argument to the established estimates $\gamma=1$.

Theorem 3. Let $0<\gamma \leq 1$. Then $R_{\gamma}$ defined in (1) satisfies a restricted weak type $(p, q)$ estimate with $p=|d| /(\gamma n)$ and $q=|d| /(\gamma(n-1))$.

Proof. Observe that when $\gamma=1 / k$ for some integer $k$, a change of variables $t \mapsto t^{k}$ reduces this theorem to the case $\gamma=1$ for some more degenerate curve. 
For all other $\gamma$, we use analytic interpolation of the restricted weak type estimates

$$
\left|\int R_{\gamma} \chi_{E}(x) \chi_{F}(x) d x\right| \lesssim|E|^{\operatorname{Re}(\gamma) \frac{n}{|d|}}|F|^{1-\operatorname{Re}(\gamma) \frac{n-1}{|d|}}
$$

between the lines $\operatorname{Re}(\gamma)=1 / k$ and $\operatorname{Re}(\gamma)=1 /(k+1)$.

Remark. The limiting case $\gamma=0$, where we define

$$
R_{0} f(x):=p \cdot v \cdot \int_{-1}^{1} f(x-h(t)) \frac{d t}{t},
$$

has long been known to be bounded on $L^{p}$ for $1<p<\infty$ as we would expect. See for example [8].

\section{Comments}

It is straightforward to show that $R_{\gamma}$ cannot be of restricted type $(p, q)$ when $\left(p^{-1}, q^{-1}\right) \notin \mathcal{T}$. One needs only test on characteristic functions of small balls about the origin and again on non-isotropic boxes, just as done in Christ [1]. The only difference is that the boxes should scale like the curve $h(t)$, i.e., they should have side lengths $\left(\delta^{d_{1}}, \ldots, \delta^{d_{n}}\right)$. The unboundedness of $R_{\gamma}$ outside $\mathcal{T}$ is also established by Tao and Wright [9], where they construct appropriate test functions for general families of curves.

The proofs presented here are independent of the endpoints of $t$-integration in (1). In particular, theorem 3 also holds for the global operators

$$
R_{\gamma} f(x):=\int_{-\infty}^{\infty} f(x-h(t))|t|^{-1+\gamma} d t
$$

as does the dual bound (but $R_{\gamma}$ is no longer bounded on $L^{1}$ or $L^{\infty}$ ).

Theorem 3 also holds in the case of curves $h(t):=\left(t^{d_{1}}, \ldots, t^{d_{n}}\right)$ where the $d_{i}$ are distinct real numbers, and $d_{i} \geq i$ for each $i$ (some modification of the region of boundedness must occur if the exponents $d_{i}$ are too small). The only new ingredients are a generalized factorization to replace (16) and an extension of Bezout's theorem to more general systems such as "fewnomials." See theorem 3 of chapter 2 in [4] for more about systems of fewnomials.

In low dimensions, the arguments presented here can be applied to $C^{\infty}$ curves of finite type (curves with $n$ linearly independent derivatives at the origin); the only change is to replace (13) by lemma 2.1 of Christ [2]. In higher dimensions, problems arise because the equation $G_{s}(\tau)=x$ can have arbitrarily many solutions $\tau$ on sets of $x$ with positive measure. What is needed is a proof that only boundedly many of these solutions lie in $\omega \subset \Omega_{k}$.

We construct an example of a "bad" $G_{s}(\tau)$ as follows. Let $\psi(t)$ be a smooth function which is identically one on the interval $[3 / 4,5 / 4]$ and vanishing outside $(2 / 3,4 / 3)$. Let

$$
\phi(t):=\sum_{k=1}^{\infty} 2^{-2^{k}}\left(t-2^{-k}\right)^{3} \psi\left(2^{k} t\right) .
$$


The function $\phi$ is $C^{\infty}$ and vanishes to infinite order at $t=0$. In addition, $\phi$ has vanishing second derivative and non-vanishing third derivative at $t=2^{-k}$ for $k \geq 1$. Consider the plane curve $h(t):=\left(t, t^{2}+\phi(t)\right)$. Following the prescription of section 4 , let $G_{\left(s_{1}, s_{2}\right)}\left(t_{1}, t_{2}\right):=h\left(t_{1}\right)+h\left(t_{1}+s_{1}\right)-h\left(t_{2}\right)-h\left(t_{2}+s_{2}\right)$. Direct computation shows that

$$
\begin{gathered}
G_{(\epsilon, 0)}\left(u-\frac{\epsilon}{2}, u\right)=\left(0, \frac{\epsilon^{2}}{2}+\phi\left(u+\frac{\epsilon}{2}\right)-2 \phi(u)+\phi\left(u-\frac{\epsilon}{2}\right)\right) \\
\operatorname{det} \frac{\partial G_{(\epsilon, 0)}}{\partial \tau}\left(u-\frac{\epsilon}{2}, u\right)=2 \frac{d \phi}{d t}\left(u+\frac{\epsilon}{2}\right)-4 \frac{d \phi}{d t}(u)+2 \frac{d \phi}{d t}\left(u-\frac{\epsilon}{2}\right) .
\end{gathered}
$$

For any $k \geq 1$, there is a solution $u_{k, \epsilon}$ of the equation $G_{(\epsilon, 0)}\left(u_{k, \epsilon}-\epsilon / 2, u_{k, \epsilon}\right)=$ $\left(0, \epsilon^{2} / 2\right)$ which approaches $2^{-k}$ as $\epsilon \rightarrow 0$; this is shown by applying the intermediate value theorem to the identity

$$
\phi\left(u+\frac{\epsilon}{2}\right)-2 \phi(u)+\phi\left(u-\frac{\epsilon}{2}\right)=\frac{\epsilon^{2}}{4} \int_{[0,1]^{2}} \frac{d^{2} \phi}{d t^{2}}\left(u+\frac{\epsilon}{2}(x+y-1)\right) d x d y
$$

and recalling that $\frac{d^{2} \phi}{d t^{2}}\left(2^{-k}\right)=0 \neq \frac{d^{3} \phi}{d t^{3}}\left(2^{-k}\right)$. It must also be that as $\epsilon \rightarrow 0$,

$$
\frac{1}{\epsilon^{2}} \operatorname{det} \frac{\partial G_{(\epsilon, 0)}}{\partial \tau}\left(u_{k, \epsilon}-\epsilon / 2, u_{k, \epsilon}\right) \rightarrow \frac{1}{2} \frac{d^{3} \phi}{d t^{3}}\left(2^{-k}\right) \neq 0
$$

(simply differentiate (19) with respect to $u$ ). We conclude that for any fixed $k \geq 1$ and any small, positive $\epsilon$, the equation $G_{(\epsilon, 0)}(\tau)=\left(0, \epsilon^{2} / 2\right)$ has a solution $\tau_{k, \epsilon}$ near the point $\left(2^{-k}-\epsilon / 2,2^{-k}\right)$; furthermore, $G_{(\epsilon, 0)}(\tau)$ is invertible near $\tau_{k, \epsilon}$ when $\epsilon$ is sufficiently small. For any natural number $N$, then, there is an $\epsilon$ and an open set of points $x$ (which includes $x=\left(0, \epsilon^{2} / 2\right)$ ) for which $G_{(\epsilon, 0)}(\tau)=x$ has at least $N$ solutions.

\section{Acknowledgments}

I would like to thank my advisor, E. M. Stein, for his instruction and guidance. I would also like to thank the referee for helpful comments and suggestions. This material is based upon work supported under a National Science Foundation Graduate Research Fellowship.

\section{References}

[1] M. Christ, Convolution, curvature, and combinatorics: a case study, Internat. Math. Res. Notices (1998), 1033-1048.

[2] - On the restriction of the Fourier transform to curves: endpoint results and the degenerate case, Trans. Amer. Math. Soc. 287 (1985), 223-238.

[3] A. Greenleaf, A. Seeger, and S. Wainger, On X-ray transforms for rigid line complexes and integrals over curves in $R^{4}$, Proc. Amer. Math. Soc. 127 (1999), 3533-3545.

[4] A. G. Khovanskiǔ, Fewnomials, trans. S. Zdravkovska, Translations of Mathematical Monographs, 88, Amer. Math. Soc., Providence, RI, 1991.

[5] D. Oberlin, A convolution estimate for a measure on a curve in $R^{4}$, Proc. Amer. Math. Soc. 125 (1997), 1355-1361. 
[6] - A convolution estimate for a measure on a curve in $R^{4}$ II, Proc. Amer. Math. Soc. 127 (1999), 217-221.

[7] I. R. Shafarevich, Basic Algebraic Geometry, trans. K. A. Hirsch, Springer-Verlag, Berlin, 1977.

[8] E. M. Stein and S. Wainger, Problems in harmonic analysis related to curvature, Bull. Amer. Math. Soc. 84 (1978), 1239-1295.

[9] T. Tao and J. Wright, $L^{p}$ improving bounds for averages along curves, J. Amer. Math. Soc. 16 (2003), 605-638.

[10] H. Weyl, The classical groups, Princeton Univ. Press, Princeton, N. J., 1946.

Mathematics Department Princeton University 311 Fine Hall Princeton, NJ 08544

E-mail address: gressman@Math.Princeton.edu 